\title{
Professor Wilfried Imrich awarded honorary doctorate at the University of Maribor
}

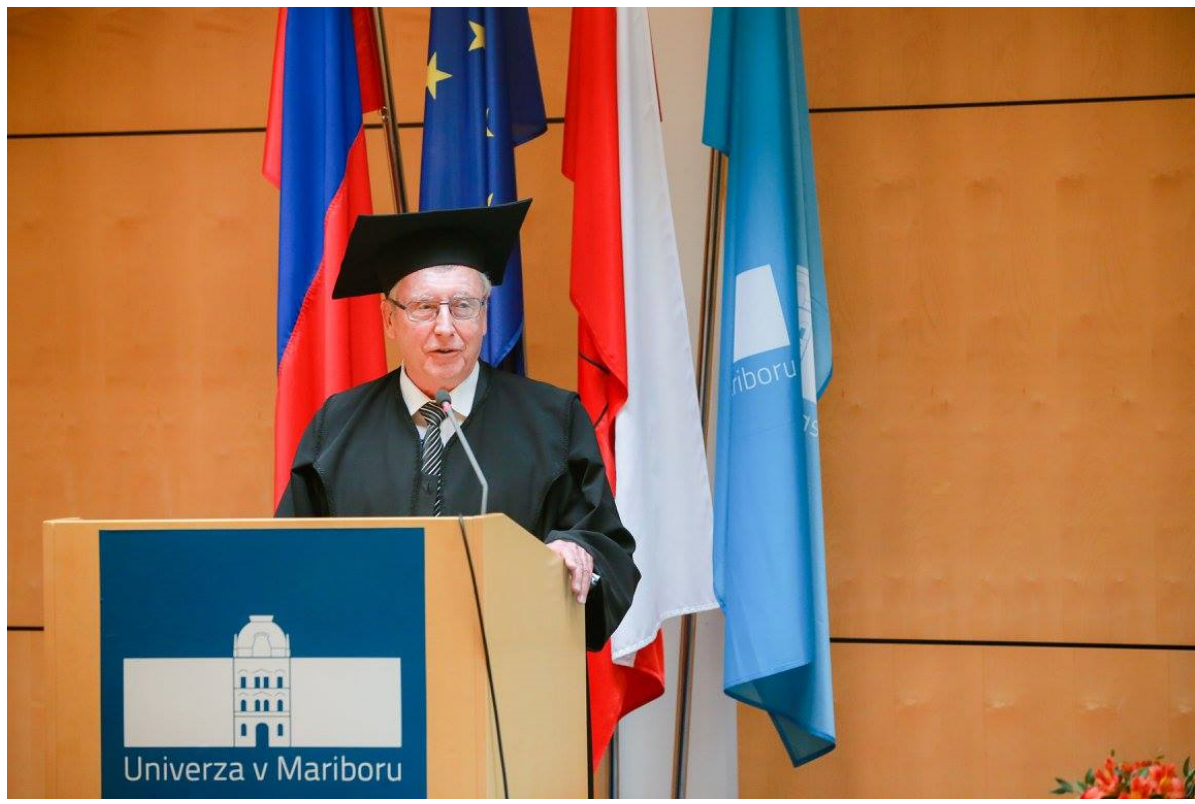

On January 31, 2018, Prof. Emer. Dr. Wilfried Imrich from the Montanuniversität Leoben, Austria, became a Honorary Doctor of the University of Maribor. The title was awarded to him for his scientific achievements and contributions to the development of the University of Maribor. The university awards this title since 1979, Wilfried Imrich is the first mathematician to receive this prestigious title. Moreover, he is the first foreign mathematician with the honorary doctor title at a Slovenian university.

The collaboration between Wilfried Imrich and the Slovenian graph theory school started when in the 1980s he established together with Tomo Pisanski the Leoben-Ljubljana seminar, which is still going on. The rest is then history. As a coincidence, the 30th Ljubljana-Leoben Graph Theory Seminar that happened in September 2017, took place for the first time in Maribor. In the last two decades, Wilfried was a frequent participant of the Seminar on discrete mathematics that is held at the Faculty of Natural Sciences and Mathematics in Maribor. He has written three books and close to fifty papers with a dozen co-authors from Maribor. The fact that at the present 16 academic descendants of Prof. Imrich have positions at the University of Maribor indicates that the award was more than deserved. 